\title{
Positive and Negative Consequences of COVID-19 Based on Perspectives of Recovered Women from COVID-19: A Qualitative Study
}

Hamed Rezakhani Moghaddam ( $\sim$ rezakhanih@gmail.com )

Khalkhal University of Medical Sciences

Vahideh Aghamohammadi

Khalkhal University of Medical Sciences

Khadijeh Nasiri

Khalkhal University of Medical Sciences

Aghil Habibi Soola

Ardabil University of Medical Sciences

Yalda Mousazadeh

Khalkhal University of Medical Sciences

\section{Research Article}

Keywords: COVID-19, qualitative research, consequences, women

Posted Date: March 7th, 2022

DOI: https://doi.org/10.21203/rs.3.rs-1329123/v1

License: (9) This work is licensed under a Creative Commons Attribution 4.0 International License. Read Full License 


\section{Abstract}

The present study was conducted to evaluate the consequences of this disease on various health dimensions of recovered women. This qualitative study was carried out using the content analysis approach in Iran. Data analysis implemented a six-stage analysis framework, using MAXQDA. Negative consequences of COVID-19 were generally categorized into five main themes including increasing selfconscious excitement, perceived social stigma, depression, changes in behavioral patterns, and reducing the level of quality of life. The positive consequences were also appeared in five categories including the development of the spiritual attitude, increasing the importance of personal health, the rise of perceived support, increasing the sense of altruism, and increasing financial savings. Based on the findings, effective supports by the government, society, and the medical staff could help to relieve the people's psychological and social stress through providing financial aid, the right information, and training.

\section{Introduction}

A new coronavirus, called modern Coronavirus-2019 in late December 2019, caused an outbreak of pneumonia from the Human (Hanan seafood market) throughout China. Following a logarithmic growth until January 23 of 2020, it spread to other countries and attracted high attention[1, 2]. The new Coronavirus, with the scientific name of SARS-CoV-2 and the disease caused by it (COVID-19 disease), has caused much concern and panic among people worldwide. World Health Organization (WHO) announced the spread of this virus as an agent of a public health emergency around the world $[1,3]$.

The new coronavirus epidemic, compared to previous common coronaviruses, is more widespread in humans, indicating a high transmission potential of the virus. However, the rate of mortality caused by new coronavirus is much lower than that of other coronaviruses such as SARS, MERS, and other viruses such as AIDS and Ebola. Almost $80 \%$ of patients who are affected by coronavirus show mild symptoms and recover at home. The affected person shows severe symptoms, including pneumonia and shortness of breath, in $14 \%$ of cases. In $5 \%$ of cases, it leads to respiratory failure, infectious shock, and dysfunction in other organs of the body $[4,5]$. The best way to cope with this disease is to avoid its infection and prevent its spread by taking protective measures and observing personal hygiene, due to the lack of effective treatment and vaccine at the present time [3]. Based on the experiences of the countries involved in the outbreak of SARS (2002) and MERS (2012), the affected people were exposed to social harms such as social exclusion, discrimination, and stigma. Also, reduced mental health was observed in people affected by this virus. They experienced the fear of their family members, lack of financial resources, lack of trust, high-risk behaviors, and anxious reactions [6].

Psychologists and other experts in this field argue that in situations like the outbreak of a disease, psychological traumas can be more dangerous than physiological traumas. The psychological effects vary, depending on the culture, cognitive disorders, mental conditions, and the media. Quarantining infected people and being away from family exacerbate the psychological traumas [7, 8]. Also, symptoms such as hypoxia, fever, cough, and drug side effects, including insomnia in people with COVID-19 can 
worsen the affected people's mental condition $[9,10]$. Valiente et al. found a high rate of mental health disorders during the confinement and detected potential predictor variables associated with experiencing both clinical levels of distress and well-being such as perception of loneliness, preexisting mental health difficulties, anxiety due to COVID-19, and substance abuse [11]. Moreover, Tull et al. found stay-at-home order was associated with greater health anxiety, financial worry, and loneliness. Furthermore, the perceived impact of COVID-19 on daily life was positively related to health anxiety, financial worry, and social support, but negatively associated with loneliness [12]. Since studies on the various consequences of this disease among people recovered from COVID-19 are relatively scarce and given a high prevalence of COVID-9 in the world and the unknown consequences of this disease in recovered women, the present study was conducted to examine the consequences of this disease on various health dimensions (physical, mental and social health) of recovered women. Being aware of the consequences of COVID-19 disease and its risk factors provides a useful indicator for the seriousness of this problem and provides an appropriate and effective public health education program to reduce its effects.

\section{Methods}

\section{Study Design}

This research was a qualitative study to examine the consequences of COVID-19 on various health dimensions (physical, mental and social health) of recovered women in Ardabil province, Iran. The data was collected over 10 weeks in June and August 2021.

\section{Setting and Samples}

In the present study, purposeful sampling of maximum variation in characteristics such as age, education, and the job was used to understand the consequences of the disease for the researcher. Twenty-six individuals were selected based on the adequacy of the generated data and analysis of them. In the present study, sampling continued until the data saturation level was reached. Participants of this study included people who recovered from the disease. Inclusion criteria were recovered females patients were registered at the health center, over the age of 18 , willing to cooperate, people who do not have cognitive disorders or severe disabilities, and able to give informed consent. women who are not ready to be interviewed were excluded from the study.

\section{Ethical considerations}

The participants who met the inclusion criteria were fully informed about the study's protocol. The study was approved by the Ethics Committee of Khalkhal University of Medical Science (IR.KHALUMS.REC.1399.02). Written consent was obtained from all participants before the interview. All individual interviews and group discussions were held at the health center and in an appropriate place. 


\section{Data collection procedure}

Individual in-depth semi-structured interviews and focus group discussions were used to collect the data. In semi-structured individual interviews, the experiences of eighteen recovered patients were scrutinized. Interviews lasted a maximum of $\mathbf{4 0}$ minutes and, if necessary, a maximum of two interviews was performed. Furthermore, two group discussion sessions were conducted with eight recovered patients. The group discussions were formed to raise issues and summarize the opinions if the results of individual interviews were contradictory or presented at a large scale. The interviews were continued until no new data could be found to expand new categories (data saturation).

Accordingly, the experienced researchers started the interview by asking for guidance and general questions. Then, the interviewer guided and continued the interview based on the stated contents and experiences, and when necessary, probing questions (such as Why? How? When?) were used.

The interviews were also recorded with the consent of the participants and then typed word by word. In the case of non-consent of participants to record the interviews, a note-taking method was used. The interviews were performed individually in a quiet environment and at the right time and place so that participants can feel comfortable. Coordination was made by obtaining the participants' consent to confirm the statements or perform the next interviews. The duration of the interview depended on the conditions of the participants. A sample of questions was:

What were the side effects or consequences of COVID-19 in your life?

The participants were also asked at the end of the interview to present solutions to overcome these barriers.

\section{Data analysis}

In the present study, the collected data were analyzed according to the Graneheim and Lundmanmethod [13]. All interviews were audio-recorded, transcribed word by word. The transcripts were read several times for general and correct understanding. All transcripts were considered as a unit of analysis. Words, sentences, and paragraphs were considered as meaning units. Meaning units were a set of words and sentences that were related to content. Then the semantic units, according to the concept hidden in them, reached the level of abstraction and conceptualization and were named with codes. The codes were compared with each other in terms of their similarities and differences and were classified under more abstract categories with a specific label. In the end, by comparing the categories with each other and deep and accurate reflection, the content hidden in the data was introduced as the theme of the study.

An initial analysis of the results was performed at the end of each session, using the notes taken. The recorded voices and the group opinions were accurately recorded and analyzed to apply the corrections, if necessary, in the next sessions. To analyze the data in the present study, MAXQDA software was used. 


\section{Data Trustworthiness}

The reliability and validity of data refer to credibility, confirmability, transferability, dependability. In the present study, we adopted the necessary strategies to observe these criteria as follows. We tried to ensure research validity by carefully selecting qualified participants, close, accurate, continuous, and long-term involvement with them, making participants involved in the process of data interpretation (reviewing by participants), using professors' opinions at different stages of study, especially in extracting themes and final interpretation.

The confirmability criterion indicates the extent to which a qualitative researcher was accurate in recording and reporting the research so that other people can follow the subject by reading these writings and achieving similar results. In the present study, the researcher tried to increase the study's confirmability as much as possible by preserving the documents in all stages of the research and providing the conditions for examining the stages and processes by others by clarifying the methodological decisions. The researcher also tried to provide the conditions for making judgments and evaluations by others to transfer results through a complete and detailed description of the environment and context, participants, and data analysis methods.

Dependability that is equivalent to reliability in quantitative studies indicates how stable and interdependent data are at the same time and under the same conditions. To ensure the dependability of the research, we provided the data to a researcher out of the research as a foreign observer to see if he had a similar understanding of the data.

Inclusion criteria: Employees with at least one year of employment history, officials or people who are willing to cooperate, and people who do not have cognitive disorders or severe disabilities.

Exclusion criteria: People who were not willing to be interviewed.

The quality assessment of the present study was done using criteria for reporting qualitative consolidated research.

\section{Results}

Twenty-six eligible women (19-75 years old ) attended the interviews. The occupational status of the participants was as follows: self-employed $(n=11)$, government employees $(n=7)$, and housewives $(n=8)$. The results of the interviews on the individual negative consequences of COVID-19 were categorized into five themes: increasing self-conscious excitement, perceived social stigma, depression, changes in behavioral patterns, and reducing the level of quality of life. Each theme contains several sub-themes that are listed in Table1. Also, positive outcomes were considered in five categories in general, including the development of the Spiritual Attitude, increasing the importance of personal health, the rise of perceived support, increasing the sense of altruism, and increase financial savings. 


\section{A) Negative Consequences of Covid 19}

\section{The first theme - Increasing self-conscious excitement}

The theme included sub-themes of increased fear and anxiety, feel guilty, stress, and anxiety.

\section{Increased fear and anxiety}

Most patients said that COVID-19 increased their level of fear and anxiety. One participant said they were so frightened that their daily lives were disrupted:

"I'm always afraid to die. It's true that I did not have an underlying disease, but I always think to myself that maybe I have heart disease, maybe I have a stomach ulcer. Maybe I have cancer and I do not know". (Participant 2)

Concerns about children's future, excessive concerns about children's health, fear of the name Covid 19, fear of being a carrier, excessive use of detergents, fear of re-infection, anxiety in other family members, a shock to the individual Death, fear of rejection in society, were among the topics frequently mentioned in interviews:

"I have no problem, I just do not want anyone to know I had a corona, because everyone is told that I had a corona and they distance themselves from me."(Participant 2)

\section{Feel guilty}

Feelings of guilt over family efforts to care for the patient, feelings of guilt over infecting others, and feelings of embarrassment caused problems in patients' daily lives:

"I have a guilty conscience because I caused my children and grandchildren to get sick. I could have followed the health tips more and distanced myself from them, but I was wrong"(Participant 2)

\section{Stress and anxiety}

COVID-19 caused nervous stress and psychological stress in patients. Some of the interviewees reported psychosomatic illnesses, insomnia, fear, and severe anxiety as a result of the disease.

\section{The second theme- Perceived social stigma}


One of the themes achieved was perceived social stigma, which included feeling excluded from society, reducing social interactions, and creating problems in the workplace.

\section{Feeling excluded from society}

The behaviors of others and acquaintances caused some patients to feel rejected by society. According to one of the participants, the behavior of his colleague in the workplace was a sign of his rejection by society:

"Even though I was in quarantine, there were signs of improvement, because my co-workers knew that I was ill, my co-worker could not accept me, for example, they called me at one of the meetings and said that there was no meeting. My colleague used a two-layer mask in the room, standing away from me, spraying the desk and computer regularly "(Participant 19)

In this regard, another participant said: "For a while, no one approached us. In the village, no one passed by our house. Everyone who came forward would shut their mouths. We were at home for a whole month" (Participant 8)

\section{Reducing social interactions}

Participants reported that their levels of social interaction decreased due to COVID-19, the distance of family members and acquaintances from the patient, disruption of family communication, the distance between the patient and his children, the distance between colleagues and the individual, limited communication with children and grandchildren, restricting family travel, the absence of the sick person in the ceremony were among the items that were expressed in this category:

"Even now, only the behavior of others bothers me. Sometimes when we talk to the neighbors, they say that you had a corona, for example, something happened and this sentence makes me nervous, so I try not to communicate too much with others"(Participant 8 )

\section{The third theme - Depression}

Depression consisting of two categories of feelings of emptiness and pessimism towards health workers was another theme that emerged in this study.

\section{Feelings of emptiness}

COVID-19 in many patients has led to feelings of emptiness in life and a sense of the futility of being in the community, said one participant: 


\section{Pessimism towards health workers}

According to some patients, the lack of facilities and manpower in medical centers had caused the treatment process to be disrupted, which was one of the reasons for their pessimism towards health workers:

"I was hospitalized and did not do anything for me, that is, I did not have many possibilities to do anything for my body. I do not have any problems now'. (Participant 6)

\section{The fourth theme - Changes in behavioral patterns}

Other themes that emerged in this study were changes in behavioral patterns after the disease. This theme also consisted of two categories including lifestyle changes and obsessive-compulsive behaviors.

\section{Lifestyle changes}

One of the most important issues mentioned by the interviewees was changes in their lifestyle due to illness, changes such as not participating in religious ceremonies, doing work through cyberspace, using medicinal plants:

"Even after recovery, our lifestyles have changed dramatically, for example, my wife does shop and doing her daily chores even though she works in a hospital. Or now all family members are trying to get the food that specialists or doctors recommend. In other words, our eating patterns at home have changed. We used to not use herbs at home, but now we do "(Participant 22)

Participants' experiences showed that having COVID-19 affected even the smallest habits of their lives, such as buying clothes:

"I have no motivation to buy other clothes like before, I used to be very fond of buying clothes, now not anymore, I used to love shopping, but now I am afraid to go shopping again". (Participant 17)

\section{Obsessive-compulsive behaviors}

Some participants claimed that obsessive-compulsive behaviors developed in them several months after COVID-19's disease. 


\section{Fifth theme - Reducing the level of quality of life}

The fifth theme consisted of two categories: creating physical problems and creating economic problems.

\section{Creating physical problems}

One of the problems reported by all participants was the development of physical problems after the disease. Problems such as weakness in the body, indigestion, premature fatigue, tachycardia, tachypnea, physical weakness, severe hair loss, difficulty hearing, and hearing were some of the cases reported by patients:

"I feel something weighing down my chest. I'm not as light and comfortable as before. I'm short of walking as I walk. I feel like my body is completely weak." (Participant 9)

"I feel like my ear has a problem. After the disease, I keep talking in my ear. The runny nose is still not getting better. I think I should try again." (Participant 1)

\section{Creating economic problems}

COVID-19 has caused financial problems for many participants, especially in those who did not have a permanent government job. One of the participants said that he even had difficulty financing the hospital and could hardly afford the leave:

"My husband was a driver and he could not leave home and go to work, which had exacerbated our economic problems. All the children had come, and l, who was in the hospital, was worried about the hospital expenses because my husband was old. And if he was going to travel, he might get sick too." (Participant 4)

\section{B) Positive Consequences of COVID-19}

\section{The first category- Development of the spiritual attitude}

Some interviewees believed that recovery from illness was like an opportunity to live again, which they believed strengthened their morale and changed their outlook on life spiritually:

"I was already preoccupied with some issues, I concluded that I should not upset anyone, the world is far away, not too long, God has allowed me to live again" (Participant 18) 


\section{The second category - Increasing the importance of personal health}

Based on the experiences of many participants, COVID-19 disease increased the importance of personal health to them. Among the positive consequences related to personal health that were obtained in the analysis of interviews are more health tips, more importance to personal health, more use of cyberspace content about health, increasing the study of scientific topics:

"Since I got the disease, I regularly wash my hands with soap and water, take a bath every day, follow the preventive ways they are called in cyberspace." (Participant 14)

\section{The third category - The rise of perceived support}

From the participants' point of view, COVID-19 disease increased their perceived social support, as they became more aware of community and family illness as a result of their disease:

"After getting sick, all the family members ask me every day how I am. My wife pays more attention to me than before" (Participant 21)

\section{The fourth category- Increasing the sense of altruism}

Some participants stated that after recovering from the disease, they decided to be careful and good at dealing with others in social interactions.

"After recovering, I realized more about the value of family, I try not to complain about anyone anymore, I do not expect from anyone, I try more to be good with other people" (Participant 13)

In this category, warming family relationships, increasing values such as family members, increasing love for family and colleagues, increasing the sense of helping others, paying more attention to family members, avoiding arguing with others, use of other patients' experiences, and the opportunity to interact more with the children were among the codes mentioned by the interviewees.

\section{The fifth category - Increasing financial savings}

According to some participants, among the positive behaviors changed in them was increasing personal savings, saving daily living, and reducing additional expenses in their lives. One of the interviewees said: 
"After recovering, I decided to save more money for the day, so that I would not borrow money from others again if I needed treatment. I think it had a positive effect on me because I did not keep my money before." (Participant 20)

\section{Discussion}

This study investigated the consequences of COVID-19 among recovered patients from this disease. We detected five negative consequences including increased self-conscious emotion, social stigma, depression, behavior pattern changes, and decreased quality of life. In addition, positive consequences included the development of the spiritual attitude, increased personal health importance, perceived support, humanity, and saving. Self-conscious emotion, as a fundamental psychological factor, plays an important role in the quality of life. Conscious emotion is linked to interpersonal. The shame and guilt feelings are the emotions that are seen after failure in interactions; these feelings are part of the emotional responsiveness of differentiation or isolation $[14,15]$. In this study, the recovered COVID-19 patients experienced severe fear, anxiety, stress, feeling guilty, some psychosomatic illnesses, and insomnia.Based on conducted studies during the COVID-19 pandemic, many people have been stated psychological problems such as stress, depression, and anxiety besides disease symptoms [16-20]. A study regarding 1-year outcomes in survivors of severe acute respiratory syndrome (SARS)and their family caregivers revealed a significant decrease in mental health among $33 \%$ of patients. In addition, $44 \%$ of patients were required to visit by psychiatry or psychology practitioners [21]. Moreover, a study in China reported Post-Traumatic Stress symptoms (PTS) in 96\% of COVID-19 patients [22]. In another study on populations affected by the COVID-19 pandemic, was expressed a high level of depression in patients who experienced COVID-19 infection [23]. In two studies on SARS-CoV-1 survivors, similar to the present study, long-term psychological effects such as fatigue, psychological distress, sleep inequality [24], and pain disorder, depression, Obsessive-Compulsive Disorder (OCD) [25] were reported. In the present study, the participants claimed to experience fears including fear of re-infection, fear of being a carrier, fear of exclusion from society, and worries about the future and health of their children. The unknown nature of COVID-19 could exacerbate these fears [26]. Other negative consequences, such as feeling guilty and annoyed and blaming others for this infection are seen [27]. In addition, most infected people stay in quarantine and so have adequate time, but according to their physical state, they cannot use their free time in a good way. So they feel lonely and aimless $[28,29]$. Consistent with our findings, in the study by Yoon Park et al., COVID-19 survivors stated concerns about transmitting others and being discriminated and that they avoided others about 1 month after discharge [30]. In a study among residents in Taiwan after the SARS epidemic, the occurrence of psychiatric disorders was $11.7 \%$ and $9.7 \%$ of participants had SARS-related discrimination [31]. In another study, similarly, there was an isolation sense and discrimination in SARS recovered nurses as they were discharged from the health center [32]. The social communication of the recovered patients was greatly reduced. The development of stigmatization and related mental health subsequent has been reported worldwide during and after public health emergencies [27, 33, 34]. In a qualitative study on individuals who have recovered from COVID-19 infection, the usual manifestations of stigma were rejection from public spaces,decrease in social 
support, decrease in income,and physical violence. Also, some factors that exacerbated the stigma were lack of information, fear of infection, the implication of police in contact tracing, segregation, and legitimization of isolation by obliged public health interventions [35]. Some participantspresentedthe feeling ofemptiness and meaningless of life.Specialists have indicated that feelings during a pandemic are very similar to grief, and individuals experience distressand emptiness, which can result in a loss of meaning in life [36, 37]. Loneliness andsocialisolation have been linked to feelings of shame, grief, emptiness, depression, clinical disease, increased cortisol rate, reduced immunity, and subsequently increased length and frequency of hospital stays $[37,38]$. In this study, some participants stated the lack of facilities and workers in medical centers lead to pessimism, dissatisfaction, and disrupted the treatment process. In a retrospective cross-sectionalthe high satisfaction level of patients from measures and policies that were performed during the COVID-19 pandemic was associated withthe small number of problemsand good surgical outcomes [39]. Implementation of appropriate facilities such as video visits insteadof in-person clinic visits can lead to patient satisfaction. High workload, especially during epidemics, may lead to a decline in the quality of services and attendant increases in patient dissatisfaction [40]. Other themes that emerged in this study were changes in behavioral patterns and lifestyle after discharge. For example, participants stated some new habits such as not participating in ceremonies, doing work using the internet, and consumption of medicinal plants. A study in the USA indicated an increase in watching TV and using the internet among adults during the pandemic [41]. In Brazil, decrease in a healthy lifestyle and an increase in behaviors with potentially negative effects on health such as the decrease in the allotted time to physical activity, increase in time watching TV or using computers mobile or tablets, intake of ultra-processed foods, and increase in smoking and alcohol intake has been found during the social restriction period [42]. The use of herbal medicines as a dietary or complementary remedy to boost immunity and prevent infection and is very common among people in various countries to reduce duration and signs of COVID-19 infection [43]. The composedtherapy of COVID-19 with Chinese and Western medicine may be useful in managing symptoms and decreasing the rate of disease development [44]. The emergence of OCD behaviors was another consequence of COVID19 in this study. In a study in the USA, about $80 \%$ of respondents reported OCD symptoms since the outbreak [45]. The study of depression and OCD in Saudi Arabia revealed some risk factors related to OCD during the new pandemic including older age, males, married individuals, employed individuals, high income, and levels of education [46]. However, the recommended behaviors in the COVID-19 such as frequent hand washing and social withdrawing are similar to some compulsive behaviors of OCD [47]. One of the complaints reported by all participants was the development of physical illness after the disease such as weakness in the body, indigestion, premature fatigue, tachycardia, tachypnea, severe hair loss, and hearing problems.Previously during SARS-CoV-1 pandemicsome physical problems such as tachycardia, bradycardia, hypotension, and cardiomegaly has been indicated [48, 49].Gastrointestinal symptoms of COVID-19 including nausea, anorexia, abdominal pain, diarrhea, and gastric bleeding have been reported in some studies [5, 50,51].COVID-19 infection has triggered financial problems based on recovered patients' perspectives.In a qualitative study in Iran, participants expressed financial concerns such as job interruptions and income loss during the COVID-19 pandemic, which was obvious among low-income jobs [52]. A qualitative study in China evaluated the psychological requirements of 
hospitalized newborns' parents during the COVID-19 pandemic and recognized five themes including an urgent request for timely up-to-date information regarding the children's status, demand for emotional and psychological assistance, decreasing the inconvenience produced by the pandemic, claim for protective information after hospital discharge, demand for financial assistance [53]. Many of the participants believed that they havefound another chance for life and appreciated their family and other people after recovering. They also understand the importance of personal hygiene and saving. COVID-19 caused some unanticipated positive results including reduced road traffic and accidents, air pollution, crime rates, improvedpublic action, behavior, sanitation, communication among families, and distance education [54]. In a study on the Spanish population, some positive effects of the COVID-19 were found. More than half of the participants informedto havelearned tovalue outdoor activities more. Other positive effects were importance of personal relationships and enjoying passing time with the family [55].

Supportsby family and friends has a great impact on improving the mental health. In a case-control study in Turkey high perceived social support had a negative association with depression symptoms among hospitalized COVID-19 patients [56].

\section{Limitations}

Due to the characteristic of a qualitative study, each interviewee's uniqueness and histories should be respected, so their specific experiences may not reflect the overall picture of psychological requirements in the stuided province.

\section{Conclusion}

For the first we found the occurrence of COVID-19 was associated with positive and negative consequences for women. Lack of right information and fear about the transmitting of COVID-19 has led to unsuitable social behaviors with patients. On the other hand, social exclusion was associated with feelings of fear, guilt, and emptiness, which led to anxiety and depression and reduced quality of life. Supports by the government, society, and health workers have the main role in decreasing mental problems in patients and providing the necessary information and training. Overall, it seems this pandemic may have long-term effects on people's health, economic and social issues that require more detailed studies. Considering religious opinions in Iran, life expectancy can be increased by strengthening spirituality. Entertaining during quarantine, family empathy, and family relationships can reduce stress and depression. The public media has an important role in reducing the stigma.

\section{Declarations}

\section{Ethics approval and consent to participate:}

The study was approved by the Ethics Committee of Khalkhal University of Medical Science, is in accordance with the Declaration of Helsinki (IR.KHALUMS.REC.1399.02). Written informed consent was 
obtained from all participants before the interview. All participant information were stored in locked file cabinets in areas with limited access.

\section{Consent for publication:}

Not applicable.

\section{Availability of data and materials:}

The data sets used and analyzed during the current study are available from the corresponding author upon making official request.

\section{Competing interests:}

We declare that we have no competing interest.

\section{Funding:}

This study is financially supported by Khalkhal University of Medical Sciences.

\section{Authors 'contributions:}

VA and HRM conceptualized, designed, conducted and analyzed the research. $\mathrm{KhN}, \mathrm{AH}, \mathrm{YM}$ helped conceptualize, design, and implement the study. The all authors drafted and contributed to the final article.

\section{Acknowledgment:}

We, with this, express our gratitude to the study participants.

\section{References}

1. Sohrabi, C., et al., World Health Organization declares global emergency: A review of the 2019 novel coronavirus (COVID-19). International Journal of Surgery, 2020.

2. SeyedAlinaghi, S., et al., Characterization of SARS-CoV-2 different variants and related morbidity and mortality: a systematic review. European Journal of Medical Research, 2021. 26(1): p. 1-20.

3. Huang, C., et al., Clinical features of patients infected with 2019 novel coronavirus in Wuhan, China. The lancet, 2020. 395(10223): p. 497-506. 
4. Heymann, D.L. and N. Shindo, COVID-19: what is next for public health? The Lancet, 2020. 395(10224): p. 542-545.

5. Pan, L., et al., Clinical characteristics of COVID-19 patients with digestive symptoms in Hubei, China: a descriptive, cross-sectional, multicenter study. The American journal of gastroenterology, 2020. 115.

6. Wang, C., et al., A novel coronavirus outbreak of global health concern. The Lancet, 2020. 395(10223): p. 470-473.

7. Brooks, S.K., et al., The psychological impact of quarantine and how to reduce it: rapid review of the evidence. The Lancet, 2020.

8. Holmes, E.A., et al., Multidisciplinary research priorities for the COVID-19 pandemic: a call for action for mental health science. The Lancet Psychiatry, 2020.

9. Xiang, Y.-T., et al., Timely mental health care for the 2019 novel coronavirus outbreak is urgently needed. The Lancet Psychiatry, 2020. 7(3): p. 228-229.

10. Mirzaei, A., H. Rezakhani Moghaddam, and A. Habibi Soola, Identifying the predictors of turnover intention based on psychosocial factors of nurses during the COVID-19 outbreak. Nursing Open, 2021.

11. Valiente, C., et al., Psychological adjustment in Spain during the COVID-19 pandemic: positive and negative mental health outcomes in the general population. The Spanish Journal of Psychology, 2021. 24.

12. Tull, M.T., et al., Psychological outcomes associated with stay-at-home orders and the perceived impact of COVID-19 on daily life. Psychiatry research, 2020. 289: p. 113098.

13. Graneheim, U.H. and B. Lundman, Qualitative content analysis in nursing research: concepts, procedures and measures to achieve trustworthiness. Nurse education today, 2004. 24(2): p. 105112.

14. Stuewig, J., et al., Shaming, blaming, and maiming: Functional links among the moral emotions, externalization of blame, and aggression. Journal of research in personality, 2010. 44(1): p. 91-102.

15. Kruse, N., The relationship between self differentiation and the levels of trust, shame, and guilt in intimate relationships. 2007: Alliant International University, Los Angeles.

16. Liu, S., et al., Online mental health services in China during the COVID-19 outbreak. The Lancet Psychiatry, 2020. 7(4): p. e17-e18.

17. Kang, L., et al., The mental health of medical workers in Wuhan, China dealing with the 2019 novel coronavirus. The Lancet Psychiatry, 2020.

18. Lai, J., et al., Factors associated with mental health outcomes among health care workers exposed to coronavirus disease 2019. JAMA network open, 2020. 3(3): p. e203976-e203976.

19. Qiu, J., et al., A nationwide survey of psychological distress among Chinese people in the COVID-19 epidemic: implications and policy recommendations. General psychiatry, 2020. 33(2). 
20. Li, S., et al., The impact of COVID-19 epidemic declaration on psychological consequences: a study on active Weibo users. International journal of environmental research and public health, 2020. 17(6): p. 2032.

21. Tansey, C.M., et al., One-year outcomes and health care utilization in survivors of severe acute respiratory syndrome. Archives of internal medicine, 2007. 167(12): p. 1312-1320.

22. Bo, H.-X., et al., Posttraumatic stress symptoms and attitude toward crisis mental health services among clinically stable patients with COVID-19 in China. Psychological medicine, 2021. 51(6): p. 1052-1053.

23. Zhang, J., et al., The differential psychological distress of populations affected by the COVID-19 pandemic. Brain, behavior, and immunity, 2020. 87: p. 49.

24. Moldofsky, H. and J. Patcai, Chronic widespread musculoskeletal pain, fatigue, depression and disordered sleep in chronic post-SARS syndrome; a case-controlled study. BMC neurology, 2011. 11(1): p. 1-7.

25. Lam, M.H.-B., et al., Mental morbidities and chronic fatigue in severe acute respiratory syndrome survivors: long-term follow-up. Archives of internal medicine, 2009. 169(22): p. 2142-2147.

26. Urooj, U., et al., Expectations, fears and perceptions of doctors during Covid-19 pandemic. Pakistan Journal of Medical Sciences, 2020. 36(COVID19-S4): p. S37.

27. Aliakbari Dehkordi, M., F. Eisazadeh, and S. Aghajanbigloo, Psychological consequences of patients with coronavirus (COVID-19): A Qualitative Study. Iranian Journal of Health Psychology, 2020. 2(2): p. 9-20.

28. Bu, F., A. Steptoe, and D. Fancourt, Who is lonely in lockdown? Cross-cohort analyses of predictors of loneliness before and during the COVID-19 pandemic. Public Health, 2020. 186: p. 31-34.

29. Joensen, L., et al., Diabetes and COVID-19: psychosocial consequences of the COVID-19 pandemic in people with diabetes in Denmark-what characterizes people with high levels of COVID-19-related worries? Diabetic Medicine, 2020. 37(7): p. 1146-1154.

30. Park, H.Y., et al., Psychological consequences of survivors of COVID-19 pneumonia 1 month after discharge. Journal of Korean medical science, 2020. 35(47).

31. Peng, E.Y.-C., et al., Population-based post-crisis psychological distress: an example from the SARS outbreak in Taiwan. Journal of the Formosan Medical Association, 2010. 109(7): p. 524-532.

32. Mok, E., et al., An exploratory study of nurses suffering from severe acute respiratory syndrome (SARS). International journal of nursing practice, 2005. 11(4): p. 150-160.

33. Singh, S., S. Bhutani, and H. Fatima, Surviving the stigma: lessons learnt for the prevention of COVID19 stigma and its mental health impact. Mental Health and Social Inclusion, 2020.

34. Lee, S., et al., The experience of SARS-related stigma at Amoy Gardens. Social science \& medicine, 2005. 61(9): p. 2038-2046.

35. Gopichandran, V. and S. Subramaniam, A qualitative inquiry into stigma among patients with Covid19 in Chennai, India. Indian Journal of Medical Ethics, 2021. 6(3): p. 1-21. 
36. De Jong, E.M., N. Ziegler, and M.C. Schippers, From shattered goals to meaning in life: life crafting in times of the COVID-19 pandemic. Frontiers in psychology, 2020. 11.

37. Berinato, S., That discomfort you're feeling is grief. Harvard Business Review, 2020. 23(03): p. 2020.

38. Pietrabissa, G. and S.G. Simpson, Psychological consequences of social isolation during COVID-19 outbreak. Frontiers in Psychology, 2020. 11: p. 2201.

39. Traiki, T.A.B., et al., Impact of COVID-19 pandemic on patient satisfaction and surgical outcomes: A retrospective and cross sectional study. Annals of Medicine and Surgery, 2020. 58: p. 14-19.

40. Ramaswamy, A., et al., Patient satisfaction with telemedicine during the COVID-19 pandemic: retrospective cohort study. Journal of medical Internet research, 2020. 22(9): p. e20786.

41. Bhutani, S. and J.A. Cooper, COVID-19 related home confinement in adults: Weight gain risks and opportunities. Obesity (Silver Spring, Md.), 2020.

42. Malta, D.C., et al., The COVID-19 Pandemic and changes in adult Brazilian lifestyles: a cross-sectional study, 2020. Epidemiologia e Serviços de Saúde, 2020. 29.

43. Panyod, S., C.-T. Ho, and L.-Y. Sheen, Dietary therapy and herbal medicine for COVID-19 prevention: A review and perspective. Journal of traditional and complementary medicine, 2020. 10(4): p. 420-427.

44. Luo, X., et al., The add-on effect of Chinese herbal medicine on COVID-19: A systematic review and meta-analysis. Phytomedicine, 2021. 85: p. 153282.

45. Wheaton, M.G., et al., How is the COVID-19 pandemic affecting individuals with obsessivecompulsive disorder (OCD) symptoms? Journal of Anxiety Disorders, 2021. 81: p. 102410.

46. AlHusseini, N., et al., Depression and Obsessive-Compulsive Disorders Amid the COVID-19 Pandemic in Saudi Arabia. Cureus, 2021. 13(1).

47. Rajkumar, R.P., Contamination and infection: what the coronavirus pandemic could reveal about the evolutionary origins of obsessive-compulsive disorder. Psychiatry research, 2020. 289: p. 113062.

48. Yu, C., et al., Cardiovascular complications of severe acute respiratory syndrome. Postgraduate medical journal, 2006. 82(964): p. 140-144.

49. Li, S.S.-I., et al., Left ventricular performance in patients with severe acute respiratory syndrome: a 30day echocardiographic follow-up study. Circulation, 2003. 108(15): p. 1798-1803.

50. Jin, X., et al., Epidemiological, clinical and virological characteristics of 74 cases of coronavirusinfected disease 2019 (COVID-19) with gastrointestinal symptoms. Gut, 2020. 69(6): p. 1002-1009.

51. Chen, N., et al., Epidemiological and clinical characteristics of 99 cases of 2019 novel coronavirus pneumonia in Wuhan, China: a descriptive study. The lancet, 2020. 395(10223): p. 507-513.

52. Akbarbegloo, M., et al., Psychosocial care experiences of patients with COVID-19 at home in Iran: A qualitative study. Health \& Social Care in the Community, 2021.

53. Fan, J., et al., A qualitative study on the psychological needs of hospitalized newborns' parents during covid-19 outbreak in China. Iranian Journal of Pediatrics, 2020. 30(2).

54. Anjankar Ashish, P., et al., Positive aspects of covid 19 pandemic: A blessing in disguise. Int. J. Res. Pharm. Sci., 2020: p. 187-191. 
55. Sandín, B., et al., Psychological impact of the COVID-19 pandemic: negative and positive effects in Spanish population during the mandatory national quarantine. J Psychopathol Clin Psychol, 2020. 25: p. 1-21.

56. Kandeğer, A., et al., Evaluation of the relationship between perceived social support, coping strategies, anxiety, and depression symptoms among hospitalized COVID-19 patients. The International Journal of Psychiatry in Medicine, 2020: p. 0091217420982085.

\section{Tables}

\section{Table 1}

Negative Consequences of Covid 19 Based on Perspectives of Recovered Patients

\begin{tabular}{|c|c|c|}
\hline Theme & Subthemes & Example statements and views \\
\hline $\begin{array}{l}\text { Increasing self- } \\
\text { conscious } \\
\text { excitement }\end{array}$ & $\begin{array}{l}\text { Increased fear and } \\
\text { anxiety, feel guilty, stress } \\
\text { and anxiety }\end{array}$ & $\begin{array}{l}\text { excessive concerns about children's health, fear of the } \\
\text { name Covid } 19 \text {, fear of being a carrier, feelings of } \\
\text { embarrassment }\end{array}$ \\
\hline $\begin{array}{l}\text { Perceived } \\
\text { social stigma }\end{array}$ & $\begin{array}{l}\text { Feeling excluded from } \\
\text { society, reducing social } \\
\text { interactions }\end{array}$ & $\begin{array}{l}\text { disruption of family communication, the distance } \\
\text { between the patient and his children, restricting family } \\
\text { travel }\end{array}$ \\
\hline Depression & $\begin{array}{l}\text { Feelings of emptiness, } \\
\text { pessimism towards } \\
\text { health workers }\end{array}$ & the lack of facilities and manpower in medical centers \\
\hline $\begin{array}{l}\text { Changes in } \\
\text { behavioral } \\
\text { patterns }\end{array}$ & $\begin{array}{l}\text { Lifestyle changes, } \\
\text { obsessive-compulsive } \\
\text { behaviors }\end{array}$ & $\begin{array}{l}\text { changes such as not participating in religious } \\
\text { ceremonies, doing work through cyberspace }\end{array}$ \\
\hline $\begin{array}{l}\text { Reducing the } \\
\text { level of quality } \\
\text { of life }\end{array}$ & $\begin{array}{l}\text { Creating physical } \\
\text { problems, creating } \\
\text { economic problems, }\end{array}$ & weakness in the body, indigestion, premature fatigue \\
\hline
\end{tabular}

Table 2

Positive Consequences of COVID-19 Based on Perspectives of Recovered Patients 
Theme

Development of the Spiritual Attitude

Increasing the importance of personal health

The rise of perceived support

Increasing the sense of altruism

Increasing financial savings

\section{Example statements and views}

an opportunity to live again, the devaluation of everyday issues and problems in life

more use of cyberspace content about health, increasing the study of scientific topics

more attention from family members

warming family relationships, increasing values such as family members, increasing love for family and colleagues

saving daily living, and reducing additional expenses 